\title{
APRENDER ENSEÑANDO. UNA PROPUESTA DE ORGANIZACIÓN DE LA ASIGNATURA CONOCIMIENTO DEL MEDIO NATURAL
}

\author{
Carmen de Lemus Varela \\ Pilar Treviño Fernández \\ Universidad de La Rioja
}

\begin{abstract}
RESUMEN
Para acercarnos al EESS es necesario adecuar los programas actuales centrándolos en el desarrollo de competencias. Aquí se presenta una posibilidad de trabajo en una asignatura troncal de las Titulaciones de Maestro de Primaria que trata de motivar al estudiante hacia el trabajo en equipo, la preparación de recursos didácticos novedosos y la reflexión ante los métodos de aprendizaje de las ciencias naturales por los universitarios a través de la lectura de textos para niños.
\end{abstract}

Palabras clave: Innovación en la Universidad, docencia, Enseñanza de las Ciencias, Formación de Maestros, didáctica universitaria, innovación pedagógica.

\section{ABSTRACT}

In order to approach "EESS" it is necessary to adapt actual programmes focusing them in the development of competences. Here a possibility of work appears in a subject from the "Primary Education Teachers Training" which tries to motivate the student towards team work, didactic resources preparation and thinking about learning methods of natural sciences by the students through the text reading for children.

Key words: Innovation at university, teaching, Primary Education teachers training, Science Teaching, university didactic, pedagogic innovation.

\section{Introducción}

Centrándonos en los presupuestos del Espacio Europeo de Enseñanza Superior presentamos un diseño de la asignatura Conocimiento del Medio Natural que, en el plan de estudios actual de la titulación de Maestro en la Universidad de la Rioja, se cursa en $1^{\circ}$ y tiene carácter troncal. 
La tarea que proponemos a los universitarios pretende fomentar el aprendizaje activo y se fundamenta en la reflexión y en el hacer. Ambos procesos se complementan, de modo que la información que obtiene el estudiante en el primero le permite hacer modificaciones en el segundo y viceversa.

Nuestros alumnos tienen la posibilidad de ejercer de profesores en una situación controlada: han de realizar el diseño de una secuencia de actividades y sus correspondientes contenidos que pueda llevarse a cabo en un aula de primaria, en torno a un tema del currículo de Conocimiento del Medio Natural. Una de las actividades consiste en la lectura de un libro de contenido científico adecuado a la edad y el nivel de los escolares, que se lleva a cabo en un centro de primaria de Logroño.

La planificación de todo el proceso se basa en la reflexión y la interacción en grupos diversos en función de la tarea a realizar.

La implicación de las profesoras encargadas de la asignatura gira en trono a la organización del trabajo en los distintos agrupamientos. Como se verá más adelante, una buena parte del tiempo se dedica a la tutoría en pequeño grupo.

Partimos de las experiencias personales de los universitarios; su propio proceso de aprendizaje y sus ideas sobre cómo se enseña nos sirven como base sobre la cual -a través de experiencias que se realizan en el aula o el laboratorio, el contacto con alumnos de primaria y el trabajo en grupo- van reestructurando sus concepciones sobre cómo se enseña y cómo se aprende.

Fases de este trabajo se han presentado en el Foro de Experiencias: Hacia la Convergencia Europea, Plan de Formación del Personal Docente e Investigador de la Universidad de La Rioja, en las convocatorias de los cursos 2004/2005 y 2006/2007.

En este artículo se describe la situación de partida, se narra el desarrollo de la experiencia y, finalmente, se hace una valoración en función de los resultados obtenidos con algunos indicadores.

\section{Punto de partida}

Parte de las actividades de la experiencia que describimos surgen en relación con el Proyecto de Divulgación y Difusión Científica de la Universidad de La Rioja 2005/2006 (Difuciencia), financiado por el Ministerio de Educación y Ciencia, Plan Nacional de I+D 2004/2007 y con la colaboración de la Biblioteca Universitaria.

El programa, denominado "Actividad de divulgación de la Ciencia dirigida a escolares de Primaria", tenía como objetivos:

- Ampliar los conocimientos de Ciencias Naturales de los escolares a través de recursos bibliográficos presentes en la Universidad. Consideramos, Lemke (2006), que los niños y niñas pueden mejorar sus conocimientos de ciencias leyendo historias asombrosas sobre el mundo natural y los avances tecnológicos.

- Estimular el interés por la cultura científica, como parte de la educación formal.

- Acercar a los escolares al entorno de la Universidad como centro donde se estudia e investiga en el ámbito de las Ciencias Naturales. 


\section{APRENDER ENSEÑANDO. UNA PROPUESTA DE ORGANIZACIÓN DE LA ASIGNATURA \\ CONOCIMIENTO DEL MEDIO NATURAL}

- Implicar a los estudiantes de Magisterio en la divulgación de la Ciencia.

- Proporcionar a los alumnos de Magisterio una experiencia real con niños.

En el aprendizaje de las ciencias el lenguaje científico se adquiere por diversas vías; una significativa es a través de las imágenes (Perales Palacios, 2006). Como todo modelo, la imagen se aproxima parcialmente al concepto, pero éste se enriquecerá si el alumno maneja algo más que su libro de texto. En este trabajo tratamos expresamente de que se utilicen las ilustraciones para fijar la atención y responder a preguntas y constatar la capacidad de argumentación y el manejo de los términos científicos (López y Jiménez, 2007) de forma que el alumno de primaria se habitúe a aprovecharlas para aprender y que el futuro maestro capte el potencial de este lenguaje de imágenes y se prepare para buscar las preguntas adecuadas y saber argumentar dando una base sólida a los razonamientos formulados por el alumnado (Pessoa de Carvalho, 2007).

La preparación y ensayo de esta actividad se llevó a cabo entre mayo y octubre de 2006. Dos becarias adscritas al Programa Difuciencia trabajaron en la selección de los libros y elaboraron un fichero de éstos agrupados por bloques: Cuerpo Humano y Salud, Animales, Plantas, Universo y Sistema Solar, Geología de la Tierra y Fósiles.

Los textos se seleccionaron atendiendo a diversos aspectos (Ford, 2006):

- Nivel de dificultad.

- Calidad de imagen y texto.

- Contenidos científicos actualizados.

- Ausencia de conceptos erróneos.

- Libros de tipo narrativo-informativo o expositivo-narrativo.

Se prepararon también dos actividades de animación: por un lado, un concurso para los escolares sobre especies protegidas y, por otro, una representación con un personaje como Charles Darwin, gran científico y lector, deteniéndonos especialmente en el viaje del Beagle para explorar, junto con nuestros alumnos las posibilidades de la historia de la ciencia para construir tanto conocimientos conceptuales como procedimentales y actitudinales (Codina, 2007).

Un colegio Público, muy cercano al campus universitario, se brindó por dos veces a llevar a sus alumnos de $3^{\circ}$ y $4^{\circ}$ de Primaria a las sesiones de "prueba" del programa que realizamos en una sala de lectura de la planta baja de la Biblioteca.

\section{Los alumnos de magisterio}

Partimos de determinadas características que presentan la mayoría de estos alumnos:

Su formación en Ciencias Naturales es deficitaria, ya que en un alto porcentaje proceden de bachilleratos que carecen de asignaturas relacionadas con las Ciencias Naturales (Lemus y Treviño, 2003). En algunos casos se trata de alumnos que ni siquiera cursaron en $4^{\circ}$ de ESO la asignatura Biología y Geología, por tratarse de una materia optativa.

Somos conscientes de estas limitaciones pero, si dedicábamos a estos aspectos conceptuales el tiempo necesario, no nos era posible desarrollar la vertiente didáctica de la asignatura, de modo que optamos por que cada grupo profundizara en los contenidos 


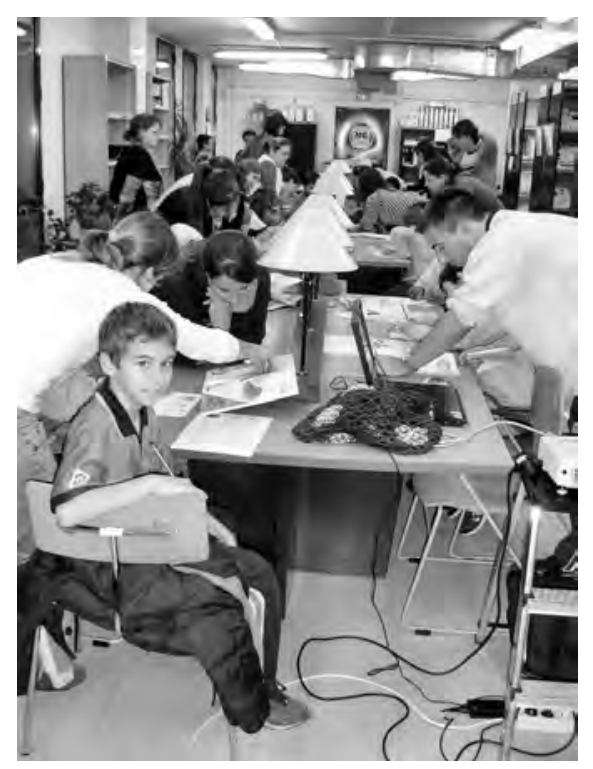

Foto 1. Alumnos de $4^{\circ}$ de Primaria leyendo en la Biblioteca de la Universidad con un grupo de estudiantes de Magisterio

científicos del tema que eligieran con independencia de que en la actividad final expusieran ante sus compañeros el contenido de su trabajo.

Desconocen todavía las concepciones de aprendizaje sobre las que fundamentar una práctica docente, debido a que esta asignatura se cursa en el primer año de la titulación. Esta cuestión nos condiciona en la medida en que para la formación en Didáctica de las Ciencias debemos seleccionar contenidos básicos que puedan integrar en su esquema conceptual.

No se han hecho preguntas acerca de cómo se enseña ni son conscientes de la dinámica de su propio proceso de aprendizaje: la perplejidad en las caras y las dificultades para expresar ideas acerca de sus vivencias escolares dan muestra de ello.

Consideran que en una situación de enseñanza-aprendizaje es más significativa la labor del profesor que la del propio alumno, a quien atribuyen un funcionamiento casi automático siguiendo las consignas del profesor. El contacto con escolares reales les hará apearse de forma definitiva de esta idea.

\section{Desarrollo de la experiencia}

La labor que proponemos a los universitarios consiste en el diseño de una secuencia de actividades y sus correspondientes contenidos que pudiera llevarse a cabo en un centro de primaria, en torno a un tema del currículo de Conocimiento del Medio Natural. Una de las actividades que deben proponer a los alumnos de la escuela es la lectura de un libro de contenido científico sobre el tema elegido, adecuado a la edad y el nivel de los escolares. 


\section{APRENDER ENSEÑANDO. UNA PROPUESTA DE ORGANIZACIÓN DE LA ASIGNATURA \\ CONOCIMIENTO DEL MEDIO NATURAL}

Como habíamos hecho en los dos cursos anteriores, el trabajo de los alumnos se estructura en torno a actividades grupales y no hay clases magistrales. Tras la presentación e información sobre el modo en que se van a impartir los 1,5 créditos durante dos horas lectivas, se divide la clase en equipos de unas 15 personas cada uno. Se trata de que estos alumnos puedan acercarse a la realidad escolar, preparando y desarrollando una sesión con alumnos de primaria de lectura y comentario de textos infantiles con contenido científico. Se ha aplicado esta metodología a un total de 146 estudiantes de $1^{\text {o }}$ de Maestro de Lengua Extranjera (LE) y $1^{\circ}$ de Maestro de Educación Musical (EM).

\section{1aㅡ fase: Elección y estudio del tema}

La elección del tema del currículo de primaria se lleva a cabo en pequeño grupo, así como la profundización en los conocimientos del bloque elegido. El grupo debe elaborar un documento con información para el profesor y pintar un mapa conceptual en el que quede plasmada la organización del tema.

Estos documentos son revisados en sesión de tutoría con las profesoras.

\section{$2^{\underline{a}}$ fase: Determinación de una secuencia de contenidos adaptada a los alumnos de pri- maria}

De nuevo, trabajo en pequeño grupo para realizar esta tarea. Se ayudan con los libros de texto de las editoriales existentes en la biblioteca. Sus experiencias como escolares son adecuadas para esta tarea. El último borrador se discute con las profesoras. De esa reunión sale la propuesta definitiva referida a contenidos.

\section{3aㅡ fase: Trabajo en la biblioteca con libros científicos para niños. Elaboración de fichas}

Para preparar la sesión con los libros, se establecen turnos de 15 personas en una sala de la Biblioteca de la Universidad; cada equipo asiste a dos sesiones que se hacen coincidiendo con su hora habitual de clase para la asignatura. Los alumnos tienen que revisar títulos para niños sobre aspectos de las Ciencias Naturales. Los libros han sido seleccionados previamente y responden a los temas de los bloques de contenidos recogidos en el programa. Entre estos libros, eligen al menos 4 sobre los que plantean preguntas por escrito que puedan aclarar y explicitar el grado de comprensión de los temas tratados.

Las profesoras revisamos y corregimos esas preguntas para la siguiente sesión. De esos juegos de cuestiones, se seleccionan las más acertadas con el criterio siempre de que sean significativas por su contenido científico, de un nivel adecuado a los alumnos a los que van dirigidas y que se puedan contestar en un tiempo breve. También se les propone que piensen y lleven otras preguntas para hacerlas de forma oral a los niños, con la intención de que se hagan idea de su capacidad de comprensión y de expresión oral, ya que creemos, como recogen López Rodríguez y Jiménez Aleixandre (2007), que se pueden mejorar las competencias argumentativas en este nivel del alumnado de Primaria. Finalmente, se prepara el material, libros y fichas en número suficiente, que se llevará al Centro escolar donde se realiza la actividad. Los cursos con los que hemos trabajado han sido los de $3^{\circ}$ y $4^{\circ}$ de Primaria. 


\section{4- fase: Realización de la actividad con los niños en los colegios}

Tras la experiencia del curso anterior, se ofertó la actividad a centros de primaria, pero trasladando la actividad a sus aulas o salas de lectura, ya que el desplazamiento de los alumnos a la Universidad suponía mayores dificultades. La actividad se realizó con doce aulas de 4 colegios.

Los estudiantes universitarios se distribuyen en el aula de Primaria de forma que atiendan entre 2 y 3 niños cada uno; les animan a leer el libro elegido en voz alta, corrigen, comentan con ellos y les presentan la ficha, que rellenan individualmente. Durante los 50 minutos que puede durar la actividad hay grupos que pueden completar de 1 a 3 libros, según lo extensos que sean. Siempre se les da una ficha para que lleven a casa o la guarde el profesor y, de las que sobran, guardamos ejemplares para comentar y comparar sus resultados. En algunos casos, hemos completado la sesión con una de las actividades de animación ya reseñadas. Cada uno de los estudiantes debe entregar un informe sobre sus impresiones a lo largo de esta la actividad.

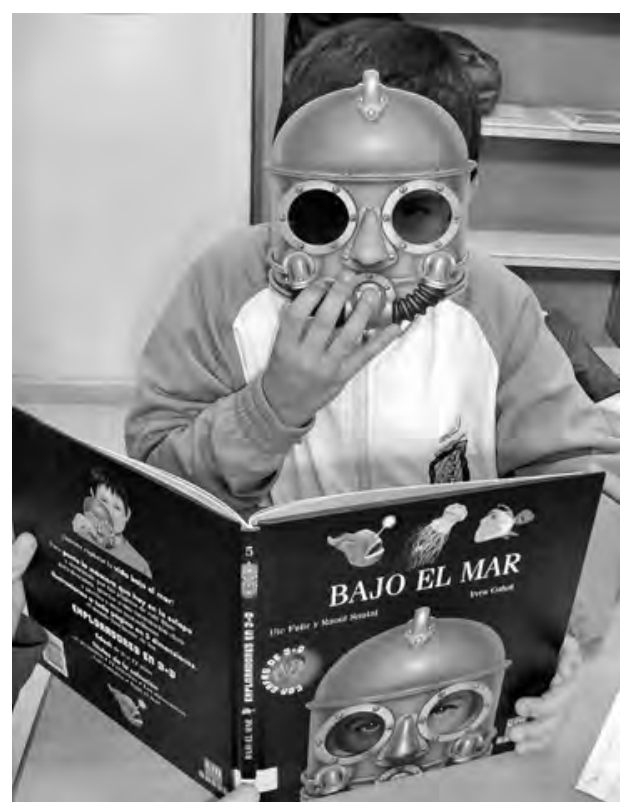

Foto 2. El "buzo"

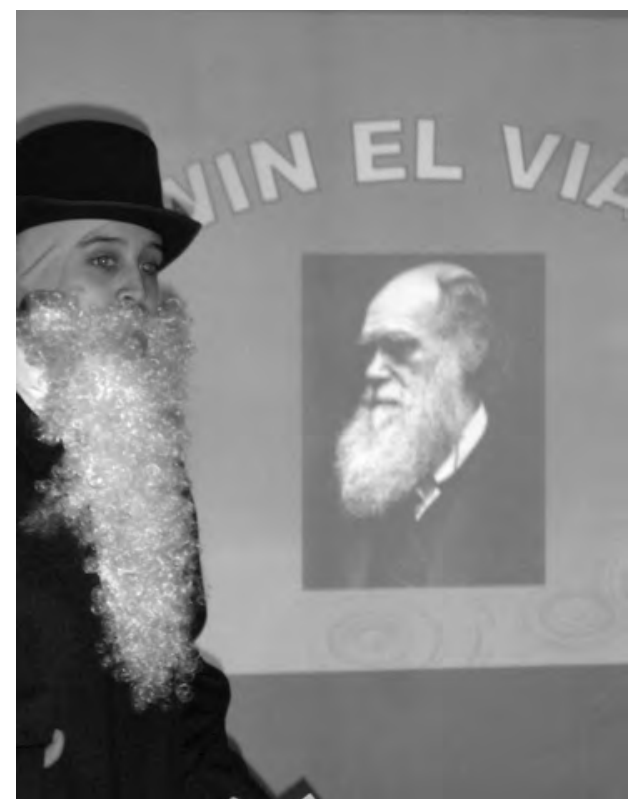

Foto 3. Alumna disfrazada representando a Darwin

\section{5aㅡ fase: Determinación de la secuencia de actividades}

Con el fin de preparar a los alumnos para esta labor, se desarrollan dos sesiones muy ligadas al quehacer de un profesor que imparta contenidos de Ciencias Naturales: una actividad de observación de materiales naturales y maquetas en el laboratorio, de 2 horas de duración, y una visita a las exposiciones de la Casa de las Ciencias del Ayuntamiento de Logroño donde se trabaja la forma de sacar rendimiento didáctico a visitas a 


\section{APRENDER ENSEÑANDO. UNA PROPUESTA DE ORGANIZACIÓN DE LA ASIGNATURA \\ CONOCIMIENTO DEL MEDIO NATURAL}

museos o salas de exposiciones. Estas dos actividades se realizan en grupo mediano (en torno a 15 personas).

El contacto con alumnos reales también ayuda a los universitarios a la hora de diseñar una secuencia de actividades, ya que tienen información sobre las capacidades de los niños de esa edad y también sobre el tipo de interacción que establecen con el profesor.

El último borrador consensuado en el pequeño grupo es analizado con las profesoras, quienes tratan de reorientar sus ideas hacia concepciones acordes con la psicología del aprendizaje y la didáctica del área.

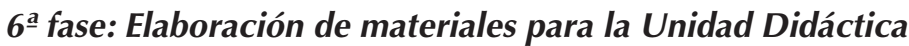

Este curso lo hemos restringido a textos y esquemas. A todos los grupos se les pide que elaboren un "libro 10", el que reúna los contenidos, ilustraciones y algunas actividades que les parezcan más adecuadas para aprender y motivar a los alumnos, basándose en la experiencia que ya han tenido con los chicos y en todos los libros que han analizado.

Los alumnos elaboran también textos o documentos en formato digital.

\section{$7^{a}$ fase: Exposición oral de trabajos}

Todos los grupos deben presentar un resumen de su trabajo y asistir a la exposición oral del resto de equipos. Para plasmar sus realizaciones pueden utilizar los medios audiovisuales o informáticos que deseen. La presentación no se considera una clase sino una exposición a otros profesionales de la educación que les pueden plantear preguntas y críticas.

\section{Temporalización}

Disponíamos de 15 horas lectivas para los alumnos ( 1 crédito teórico y 0,5 prácticos de laboratorio), que se han transformado en 11 horas presenciales obligatorias (una reducción de un 22,5\%). La asistencia a clases en gran grupo se ha cambiado por trabajo en agrupamientos más reducidos: grupo mediano de unos 15 alumnos y pequeño grupo de entre 4 y 5 personas.

La distribución del tiempo que dedican los alumnos a esta asignatura con presencia de las profesoras figura en la tabla nำ1. A esto hay que añadir el trabajo que realizan de discusión en pequeño grupo para elaborar las tareas propuestas.

Tabla 1. Distribución del trabajo presencial obligatorio de los alumnos

\begin{tabular}{lll}
\hline Actividad & Tiempo & Agrupamiento \\
\hline Presentación de la asignatura & 2 horas & Gran grupo \\
\hline Trabajo en la biblioteca & 2 horas & Grupo mediano \\
\hline Actividad en el colegio & 1 hora & Gran grupo \\
\hline Asistencia a tutorías & 2 horas (varias sesiones) & Grupo pequeño \\
\hline Prácticas de laboratorio & 2 horas & Grupo mediano \\
\hline Visita a la Casa de las Ciencias & 1 hora & Gran grupo \\
\hline Presentación oral de trabajos & 1 hora & Grupo mediano \\
\hline
\end{tabular}


En cuanto al trabajo de atención directa a los alumnos por parte de las 2 profesoras (Tabla $\mathrm{n}^{\circ}$ 2) se distribuye de la siguiente forma: las 50 horas lectivas, resultantes de las 20 de teoría y 30 de prácticas de laboratorio (el número de alumnos permite formar 6 grupos de prácticas) en conjunto para las dos titulaciones, pasan a 112 horas según se desglosa en la tabla $n^{\circ}$ 2. Esto supone que, de las horas de tutorías correspondientes a las 10 semanas en que se imparte la materia, 60 de la profesora a tiempo completo y 30 de la profesora a tiempo parcial, hay que dedicar el 61,5\% a trabajar en grupos reducidos, ya que se han formado 17 pequeños grupos en LE y 18 en EM.

Tabla 2. Distribución del trabajo presencial de las profesoras

\begin{tabular}{lll}
\hline Actividad & Agrupamiento & № de horas \\
\hline Presentación de la asignatura & Gran grupo (LE+EM) & 4 horas \\
\hline Trabajo en la biblioteca & Grupo mediano (6 grupos) & 12 horas \\
\hline Actividad en el colegio & Gran grupo (2 profesoras) & 4 horas \\
\hline Asistencia a tutorías & Grupo pequeño (18+17) & 70 horas \\
\hline Prácticas de laboratorio & Grupo mediano (6 grupos) & 12 horas \\
\hline Visita a Casa de las Ciencias & Gran grupo (2 profesoras) & 4 horas \\
\hline Presentación oral de trabajos & Grupo mediano & 6 horas \\
\hline
\end{tabular}

En una encuesta realizada a los alumnos al terminar el trabajo, se les pide que estimen el tiempo que han dedicado al trabajo en grupo al margen de las horas de clase. Sus opiniones se muestran en la tabla $n^{0} 3$.

Tabla 3. Estimación, por parte de los alumnos, de las horas de trabajo, aparte de las de asistencia obligatoria

\begin{tabular}{|c|c|c|}
\hline $\begin{array}{l}\text { Horas estimadas para hacer } \\
\text { el trabajo en grupo }\end{array}$ & Lengua Extranjera (LE) & $\begin{array}{l}\text { Educación } \\
\text { Musical (EM) }\end{array}$ \\
\hline Entre 10 y 15 horas & $26 \%$ & $48,5 \%$ \\
\hline Entre 15 y 20 horas & $37 \%$ & $37,1 \%$ \\
\hline Más de 20 horas & $37 \%$ & $14,4 \%$ \\
\hline
\end{tabular}

\section{Evaluación y análisis de resultados}

Para poder valorar la experiencia disponemos de herramientas objetivas, como son las calificaciones, y subjetivas, fundamentadas en las impresiones de las profesoras. En primer lugar, analizaremos las calificaciones obtenidas por los universitarios en esta

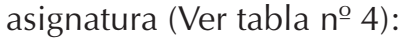


Tabla 4. Calificaciones de la asignatura

(entre 0 y 3,5 puntos, menos de 2 puntos supone suspender la asignatura)

\begin{tabular}{lccccccc}
\hline Titulación & $\begin{array}{c}\text { № } \\
\text { matriculados }\end{array}$ & $\begin{array}{c}\% \\
\text { presentados }\end{array}$ & $\begin{array}{c}\% \\
\text { aprobados } \\
\text { sobre } \\
\text { matrículados }\end{array}$ & $\begin{array}{c}\% \\
\text { aprobados } \\
\text { sobre }\end{array}$ & $\begin{array}{c}\% \\
\text { calificados } \\
\text { entre 2 } \\
\text { y 2,5 }\end{array}$ & $\begin{array}{c}\% \\
\text { calificados } \\
\text { entre 2,5 } \\
\text { y 3 }\end{array}$ & $\begin{array}{c}\text { \% } \\
\text { calificados } \\
\text { con más } \\
\text { de 3 }\end{array}$ \\
\hline $\mathrm{LE}$ & 62 & $75,8 \%$ & $61,2 \%$ & $80,85 \%$ & $50 \%$ & $23,65 \%$ & $26,35 \%$ \\
\hline $\mathrm{EM}$ & 83 & $77,1 \%$ & $56,62 \%$ & $73,43 \%$ & $53,2 \%$ & $19,15 \%$ & $27,65 \%$ \\
\hline
\end{tabular}

Se han presentado a evaluación 64 alumnos en EM (número total de matriculados 83) y 47 de LE (número total de matriculados 62). Los porcentajes de presentados sobre matriculados se explican porque hay estudiantes que no asisten a clase y se han desentendido de la asignatura, abandonos, etc. además de personas que no se presentan a evaluación por considerar que no alcanzan el nivel adecuado.

La diferencia de 7 puntos de los presentados en LE sobre los de EM puede deberse al hecho de que estos últimos cursaron la asignatura en fechas más próximas a los exámenes de junio y la acumulación de trabajo hizo que algunos desistieran.

La parte de la asignatura que impartimos supone el 35\% de la calificación total de la materia a sumar con la parte de Materia y Energía, que supone el $75 \%$ restante. Exigimos a nuestros alumnos obtener un mínimo de 2 puntos (5,7 sobre 10) para poder realizar la adición.

La calificación se realiza analizando el dossier que presentan con las actividades señaladas anteriormente sobre el tema elegido:

- Contenidos para el profesor, incluyendo un mapa conceptual.

- Secuencia de actividades.

- Libro 10.

- Documento sobre la sesión en el colegio.

Como puede verse, el porcentaje de alumnos aprobados sobre los presentados es elevado ( $81 \%$ en LE y $73 \%$ en EM), luego podemos pensar que estamos en la buena dirección. Entre los suspendidos se encuentra algún grupo que realizó el trabajo, pero que no vino a las revisiones en tutoría, de modo que sus documentos no cumplieron los requisitos exigidos.

Una vez realizada la corrección de todos los trabajos, se aprecia en las calificaciones que el curso de Lengua Extrajera ha obtenido puntuaciones algo más altas que el grupo de E. Musical. Se detecta cierta correlación entre el tiempo dedicado al trabajo y la calidad del mismo (Ver tabla $n^{\circ} 3$ ).

La distribución de las calificaciones dista de ser normal; está escorada hacia la zona de las puntuaciones más altas. En torno a la mitad de los alumnos obtienen entre 5,7 y 7 puntos sobre 10 ; alrededor de un $21 \%$ tienen entre 7 y 8,5 ; y un $26,5 \%$ tienen sobre- 
saliente. Alrededor de un 50\% de los universitarios tienen más de 7, y creemos que esto es un buen resultado.

Tanto los universitarios como los alumnos de Primaria se sienten muy motivados con la experiencia de la lectura de libros: los pequeños preguntan que cuándo volvemos y los estudiantes se ofrecen a hacerlo otra vez. De hecho, concurrimos, invitados por los centros, a dos Semanas Culturales. Se han manejado unos 80 textos infantiles y realizado 100 fichas -se hace más de una para el mismo libro- que se ponen a disposición del Colegio que nos acoge para hacer la actividad. Es una manera de colaborar a la renovación de las bibliotecas escolares.

En cuanto a los trabajos se observa una progresiva mejora en las presentaciones, cada promoción evidencia más soltura en el manejo de la tecnología informática pero tienen las dificultades de siempre para decidir sobre unos contenidos y actividades adecuados al nivel educativo y que sean coherentes con un planteamiento científico.

Un inconveniente que siempre está presente es la dificultad de un modelo que exige el trabajo en grupo y unas determinadas asistencias. Aunque para las tutorías se da mucha flexibilidad al tratarse de varios grupos (17 y 18 respectivamente), se hace complicado establecer unos horarios. La casuística de los alumnos que trabajan, viven fuera de la ciudad, tienen hijos, compatibilizan asignaturas de distintos cursos, asisten a la Escuela de Idiomas o al Conservatorio, etc, se va incrementando al ser los grupos más numerosos.

Podría pensarse que, si en las sesiones de tutoría dirigimos el trabajo hacia los baremos que hemos establecido para calificar, es fácil que tengan buenas notas. Podemos asegurar que esas tutorías han sido agotadoras: en primer lugar, por la gran cantidad de datos que traían para analizar y que era preciso desbrozar y delimitar para que se ajustaran a un trabajo para escolares en las condiciones propuestas; en segundo, porque han sido ratos de intensas discusiones: estaban motivados por el contacto con los niños y querían hacerlo bien.

Hemos disfrutado en estas reuniones; en primer lugar, por el entusiasmo que mostraban los universitarios pero, especialmente, cuando proponiendo ejemplos y contraejemplos, argumentando, comparando con situaciones en sus clases.... Ilegaban al punto de comprender cuestiones que parecen tan evidentes como que los alumnos aprenden con lo que hacen, más que con lo que oyen, o la importancia que tiene para los escolares interactuar con el mundo real para poder alcanzar conceptos de mayor grado de abstracción. Esa expresión en el rostro y el brillo en la mirada que viene después, cuando comprenden el alcance de esa nueva idea y prevén los cambios que tendrán que realizar en su mente y en su trabajo, son para nosotras una gran satisfacción.

Para poder conocer la opinión de nuestros alumnos acerca de algunos aspectos de esta asignatura se elaboró la encuesta de valoración que aparece en el anexo. Hemos conseguido 60 respuestas, lo que supone el 54 \% respecto al número de personas presentadas en las dos especialidades.

En cuanto a la estimación del tiempo que han dedicado al trabajo en grupo, al margen del presencial en la Universidad (Ver tabla $n^{\circ} 3$ ), existen diferencias entre el número de horas empleadas en las dos especialidades. Este hecho puede explicarse si se considera que el grupo de LE tuvo más tiempo, ya que las clases se dieron entre febrero y 
abril, mientras que en EM ha estado más limitado por la proximidad de las evaluaciones del final del cuatrimestre. Coincidimos con el criterio expuesto por Garmendia M., Guisasola, J., Barragués, J.I. y Zuza, C. (2006) en que los resultados son mejores si los alumnos tienen las evaluaciones espaciadas durante el curso y no tan concentradas durante los exámenes finales.

Considerando que en los dos cursos presentan los mismos porcentajes en torno a las 15-20 horas de trabajo fuera de clase, estimamos que el trabajo total del alumno estaría entre 25 y 30 horas y podría equipararse a 1 crédito ECTS.

El segundo bloque de preguntas va encaminado a que opinen sobre cómo contribuyen a su formación como maestros las actividades más significativas que han realizado.

La valoración que realizan los alumnos sobre la idoneidad de las actividades propuestas es positiva en términos generales: en todos los casos, excepto en la salida a una exposición, el grueso de las respuestas se encuentran entre las puntuaciones 4 y 5 , como puede verse en la tabla 6 .

Los dos grupos coinciden en considerar la preparación de la actividad para el colegio y la realización de las actividades con alumnos de Primaria como lo más significativo en su formación como futuros docentes. Ciertamente es a lo que han dedicado más energía porque les hacía ilusión tener contacto con niños y necesitaban hacer las cosas bien.

Dan menos valor a hacer una visita a una exposición en la invirtieron un esfuerzo menor, porque tenían otros focos que exigían más su atención. Habrá que replantearla para futuros cursos.

Si comparamos los datos de las dos titulaciones, encontramos que el grupo de EM ha dado, en todos los apartados que se consideran, porcentajes más altos, esto a pesar de ser el grupo que tuvo menos tiempo para hacer el trabajo.

Tabla 5. Valoración de la actividad en relación a su formación como futuros docentes (entre 0 y 5 )

\begin{tabular}{lrrrrr}
\hline Titulación/calificación & $\mathbf{1}$ & $\mathbf{2}$ & $\mathbf{3}$ & $\mathbf{4}$ & $\mathbf{5}$ \\
\hline $\begin{array}{l}\text { Preparación de la actividad } \\
\text { con niños de Primaria }\end{array}$ & & & & & \\
\hline L. Extranjera & $0 \%$ & $8,0 \%$ & $4,0 \%$ & $32 \%$ & $56 \%$ \\
\hline Ed. Musical & $2,9 \%$ & $0 \%$ & $5,7 \%$ & $31,4 \%$ & $60,0 \%$ \\
\hline Asistencia al colegio & & & & & \\
\hline L. Extranjera & $0 \%$ & $8,0 \%$ & $12,0 \%$ & $16 \%$ & $64 \%$ \\
\hline Ed. Musical & $0 \%$ & $0 \%$ & $8,7 \%$ & $22,8 \%$ & $68,5 \%$ \\
\hline Clases prácticas & & & & & \\
\hline L. Extranjera & $0 \%$ & $8,0 \%$ & $28,0 \%$ & $36,0 \%$ & $28,0 \%$ \\
\hline Ed. Musical & $0 \%$ & $5,8 \%$ & $11,4 \%$ & $31,4 \%$ & $51,4 \%$ \\
\hline
\end{tabular}


Tabla 5. Valoración de la actividad en relación a su formación como futuros docentes (entre 0 y 5) (continuación)

\begin{tabular}{lrrrrr}
\hline Titulación/calificación & $\mathbf{1}$ & $\mathbf{2}$ & $\mathbf{3}$ & $\mathbf{4}$ & $\mathbf{5}$ \\
\hline Tutorías con las profesoras & & & & & \\
\hline L. Extranjera & $0 \%$ & $20,8 \%$ & $16,6 \%$ & $37,6 \%$ & $25 \%$ \\
\hline Ed. Musical & $3 \%$ & $3 \%$ & $30 \%$ & $39,3 \%$ & $24,7 \%$ \\
\hline Salida a exposición & & & & & \\
\hline L. Extranjera & $14,2 \%$ & $19 \%$ & $33,6 \%$ & 14,2 & $19 \%$ \\
\hline Ed. Musical & $9 \%$ & $6 \%$ & $47,6 \%$ & $31,6 \%$ & $5,8 \%$ \\
\hline
\end{tabular}

Tabla 6. Valoración de la actividad en relación a su formación como futuros docentes (columnas 4+5)

\begin{tabular}{lcc}
\hline & Lengua Extranjera (4+5) & Educación Musical (4+5) \\
\hline Preparación actividad & $88 \%$ & $91,4 \%$ \\
\hline Asistencia al colegio & $80 \%$ & $91,3 \%$ \\
\hline Clases prácticas & $64 \%$ & $82,8 \%$ \\
\hline Tutorías con las profesoras & $62,6 \%$ & $64 \%$ \\
\hline Salida a exposición & $23,2 \%$ & $37,4 \%$ \\
\hline
\end{tabular}

Les pedimos que señalen los aspectos en los que encontraron más dificultades (ver tabla 7) y el dato que señalábamos acerca de la escasa formación científica de los alumnos se ve confirmado puesto que tuvieron que dedicarle bastante tiempo en el grupo y también en las revisiones en tutoría.

Los porcentajes de los dos grupos confirman también las carencias en la formación didáctica de los estudiantes y por ese motivo aparecen porcentajes altos al acometer la secuencia de actividades. Resulta chocante que personas jóvenes e inquietas asuman con pasmosa facilidad que el maestro manda y el alumno obedece, o que las actividades son esas preguntas que van numeradas de 1 al 30 al final de cada tema en el libro de texto.

Hemos tenido muchas sugerencias de mejora del planteamiento de la asignatura; al dominar las que piden más interactividad con niños y más tiempo de preparación de esta experiencia, coinciden con los resultados obtenidos por Aramendi P., Ayerza, M. y Bujan, K., (2004), lo que nos hace pensar que el trabajo en este sentido merece la pena. Otras demandas se refieren a la posibilidad de más tutorías y materiales para hacer los trabajos así como más tiempo para esta parte de la asignatura. 
Tabla 7. Dificultades encontradas en la realización del trabajo

\begin{tabular}{lcc}
\hline & Lengua Extrajera & Educación Musical \\
\hline Programar los contenidos & $28,3 \%$ & $16,6 \%$ \\
\hline Diseñar las actividades & $13,3 \%$ & $25 \%$ \\
\hline Coordinarse con las personas del grupo & $8,3 \%$ & $1,6 \%$ \\
\hline Hacer el "libro 10" & $8,3 \%$ & $6,6 \%$ \\
\hline Exponer el tema & $3,3 \%$ & $3,3 \%$ \\
\hline Adaptarse al nivel de Primaria & $1,6 \%$ & $0 \%$ \\
\hline $\begin{array}{l}\text { Otras (recopilar información, no poder } \\
\text { sacar los libros utilizados de la Biblioteca, } \\
\text { poco tiempo...) }\end{array}$ & $1,6 \%$ & $1,6 \%$ \\
\hline
\end{tabular}

\section{Conclusiones}

Esta forma de organización de la asignatura alcanzaría mejores resultados en el segundo año de carrera, cuando el estudiante tenga su marco conceptual más perfilado en cuestiones como la psicología del aprendizaje o la didáctica general.

Podría encontrarse alguna ventaja para los universitarios el hecho de tener una experiencia de este tipo en el primer curso: les hace tomar contacto con la dimensión de su trabajo, vislumbran su alcance, los esfuerzos que exige o las insatisfacciones que puede producir. Puede servir para que se reafirmen en su decisión y les sirva de acicate para avanzar en sus estudios, o justo para lo contrario.

Sería importante no acumular las pruebas de evaluación en un corto período de tiempo porque esto les obliga a procesar información a toda prisa, sin dejar el tiempo necesario para realizar las actividades, las reflexiones y las discusiones que exigen algunos aprendizajes.

Es innegable que hacer que los estudiantes de Magisterio tengan contacto directo con escolares redunda en beneficio de su formación: en primer lugar, por lo que supone de motivación y, en segundo, porque les obliga a poner mecanismos en marcha: imaginación, ingenio, esfuerzo... que, de otro modo, un trabajo académico no podría movilizar.

Nos parece importante que los alumnos ejerciten las capacidades que su futuro desempeño les va a exigir: planificar, trabajar en grupo, hablar en público o hacer una exposición oral ante colegas. Los alumnos se lo han tomado muy en serio y han respondido en consecuencia.

En líneas generales, los alumnos han trabajado con ganas. Se trata de una propuesta que les ha motivado. El hecho de tener contacto con alumnos "de verdad" es un gancho importante, pero también se han implicado en las otras actividades que no iban a ser aplicadas en el colegio. 
Las profesoras hemos dedicado muchas horas y esfuerzo a esta tarea, pero compensa al ver que los estudiantes trabajan con ilusión y hacen propuestas para conseguir una escuela "emocionante" para alumnos y profesores.

\section{Agradecimiento}

A Montse y Raquel, becarias del programa Difuciencia, por sus ideas y creatividad.

A la dirección y profesores los Centros educativos participantes.

A la directora y al personal de la Biblioteca de la Universidad de La Rioja.

A nuestros alumnos y alumnas por su entusiasmo.

\section{Bibliografía}

Aramendi, P., Ayerza, M. y Bujan, K. (2004). La incidencia de la Convergencia Europea y la nueva concepción de los créditos"ECTS" en los procesos de Enseñanza-Aprendizaje en la Universidad. Organización y Gestión Educativa, 6, 31-35.

Buendía, L. y Olmedo, E. M. (2000). Estrategias de aprendizaje y procesos de evaluación en la Educación Universitaria. Bordón, 52 (2), 151-163.

Codina, J.C. (2007). Viaje a la Biología con el "Beagle". Alambique, 53, 119-124.

Ficha técnica de propuesta de Título Universitario de grado de Maestro de Educación Primaria según RD 55/2005, de 21 de enero.

Ford, D. J., Brickhouse, N.W., Lotttero-Perdue, P y Kittleson, J. (2006). Elementary Girl's Science Reading at Home and School. Wiley InterScience, 7 de febrero de 2006. (www.interScience.wiley.com)

Garmendia, M., Guisasola, J., Barragués, J I. y Zuza, C. (2006). ¿Cuánto tiempo necesita invertir el alumnado para el aprendizaje de una asignatura? Estimación de los créditos ECTS de una asignatura de primer curso de Ingeniería. Revista Interuniversitaria de Formación del Profesorado, 20 (3), 271-286.

Guisasola, J., Zubimendi, J L., Almudí, J M. y Ceberio, M. (2007). Propuesta de enseñanza en cursos introductorios de física en la universidad, basada en la investigación didáctica: siete años de experiencia y resultados. Enseñanza de las Ciencias, 25 (1), 91-103.

Lara Guerrero, J. (1997). Estrategias para un aprendizaje significativo- constructivista. Enseñanza de las Ciencias, 15, 29-50.

Lemke, J. L. (2006). Investigar para el futuro de la Educación Científica: nuevas formas de aprender, nuevas formas de vivir. Enseñanza de las Ciencias, 24 (1), 5-12.

Lemus, M. C. y Treviño, M. P. (2003). Actividad didáctica para la formación inicial del profesorado en la divulgación del Patrimonio Paleontológico (exposiciones, museos y yacimientos referidos a Dinosaurios y otros reptiles mesozoicos). Ciencias de la Tierra, 26, 413-422. Ediciones del Instituto de Estudios Riojanos. Logroño.

López Rodríguez, R. y Jiménez Aleixandre, M. P. (2007). ¿Podemos cazar ranas? Calidad de los argumentos de alumnado de primaria y desempeño cognitivo en el estudio de una charca. Enseñanza de las Ciencias, 25 (3), 309-323. 
Perales Palacios, F. J. (2006). Uso y abuso de la imagen en la Enseñanza de las Ciencias. Enseñanza de las Ciencias, 24 (1), 13-30.

Perkins, J. (2001). Educación a distancia: cuando lo tradicional se torna revolucionario. Revista Bitácora, 16.

Pessoa de Carvalho, A. M. (2007). Enseñar física y fomentar una enculturación científica. Alambique, 51, 66-75.

\section{ANEXO}

\section{Encuesta de evaluación de la asignatura}

1. Haga una estimación del tiempo que ha empleado en elaborar este trabajo aparte de la asistencia a las sesiones obligatorias :

\begin{tabular}{|l|l|}
\hline Entre 5 y 10 horas & \\
\hline Entre 10 y 15 horas & \\
\hline Más de 15 horas & \\
\hline
\end{tabular}

2. Valore de 1 (mínimo) a 5 (máximo) las actividades programadas considerando su contribución a la formación docente

\begin{tabular}{|c|c|c|c|c|c|}
\hline & 1 & 2 & 3 & 4 & 5 \\
\hline Preparación de la actividad con & & & & & \\
\hline Realización de la actividad en e & & & & & \\
\hline Clases prácticas & & & & & \\
\hline Tutorías & & & & & \\
\hline Salida a la Casa de las Ciencias & & & & & \\
\hline
\end{tabular}


3. Cite las dificultades que ha encontrado en la realización del trabajo

\begin{tabular}{|l|l|}
\hline Programar los contenidos & \\
\hline Coordinación entre los miembros del grupo & \\
\hline Diseñar las actividades & \\
\hline $\begin{array}{l}\text { Adaptar contenidos y actividades al nivel } \\
\text { de Primaria elegido }\end{array}$ & \\
\hline Exponer el tema & \\
\hline Hacer el "libro 10" & \\
\hline Otras & \\
\hline
\end{tabular}

4. Sugerencias para mejorar esta parte de la asignatura

1.

2.

3.

4. 